\title{
A variable star survey of the open cluster M 37
}

\author{
L. L. Kiss ${ }^{1,4}$, Gy. M. Szabó ${ }^{1}$, K. Sziládi ${ }^{1,4}$, G. Fürész ${ }^{1}$, K. Sárneczky ${ }^{2,4}$, and B. Csák ${ }^{1,3}$ \\ 1 Department of Experimental Physics and Astronomical Observatory, University of Szeged, Dóm tér 9., \\ 6720 Szeged, Hungary \\ 2 Department of Physical Geography, ELTE University, 1088 Budapest, Ludovika tér 2., Hungary \\ 3 Department of Optics \& Quantum Electronics, University of Szeged, POB 406, 6701 Szeged, Hungary \\ ${ }^{4}$ Guest Observer at Konkoly Observatory
}

Received 19 March 2001 / Accepted 5 July 2001

\begin{abstract}
A CCD photometric study of the dense galactic open cluster M 37 is presented and discussed. The majority of the analysed data are time-series measurements obtained through an $R_{\mathrm{C}}$ filter. The observations were carried out on seven nights between December 1999 and February 2000, and have led to the discovery of 7 new variable stars in the field. Three of them have been unambiguously identified as W UMa-type eclipsing binaries, while two more are monoperiodic pulsating stars, most probably high-amplitude $\delta$ Scuti-type variables. The remaining two stars seem to be long-period eclipsing binaries without firm period determination. Johnson $B$ and $V$ frames have been used to construct a new colour-magnitude (CM) diagram of the cluster, and to find the locations of the new variable stars. The pulsating variables are most likely background objects. The CM diagram is fitted with recent isochrones yielding the main parameters of the cluster.
\end{abstract}

Key words. open clusters and associations: general - open clusters and associations: individual: M 37 stars: variables: general - stars: variables: $\delta$ Sct - stars: binaries: eclipsing

\section{Introduction}

Variable stars in open clusters provide an important tool to test theoretical predictions concerning stellar structure and evolution, because the same distance, age, initial chemical abundance and interstellar reddening can be assumed for all the stars in the same cluster. Moreover, studying cluster variables is one of the most effective and productive uses of small and moderate size telescopes. Simultaneous photometry of hundreds of stars enables very accurate light curves to be obtained. In the recent years, substantial development has been made in this area, owing to the importance of, for instance, studying stellar pulsation (Frandsen \& Arentoft 1998) or evolution of close binaries (Kałużny \& Rucinski 1993) in open clusters. Evolutionary effects make different types of variable stars appear in specific range of cluster ages, therefore, it is highly desirable to make observations of as many clusters with different ages as possible. The function of this paper is to contribute to this issue with new observations of the intermediate-age open cluster M 37.

M $37\left(=\right.$ NGC $2099=\mathrm{C} 0549+325, \quad \alpha_{2000}=$ $\left.05^{\mathrm{h}} 52^{\mathrm{m}} \cdot 3, \delta_{2000}=+32^{\circ} 33^{\prime}\right)$ is a very rich open cluster located in the low galactic latitude $(b=+3.09)$ region in the

Send offprint requests to: L. L. Kiss, e-mail: 1.kiss@physx.u-szeged.hu constellation Auriga. Although it is among the brightest open clusters in the northern sky, the observational material in the literature is quite scanty. The last extensive photometry is that of West (1967) who presented photographic $U B V$ measurements for 930 stars in the cluster. The main sequence is rather broad and the available proper motions (Jefferys 1962; Upgren 1966) do not enable a clear distinction between the member and nonmember stars. Robin (1982) published electronographic plate measurements for selected stars in the cluster in the $U B V$ bands for calibration purposes. The fifth catalogue of Lyngå (1987) lists the following cluster parameters: $m-$ $M=11^{\mathrm{m}} \cdot 63, E(B-V)=0 .{ }^{\mathrm{m}} 31$ and $\log t=8.30$. The most recent update of these parameters was given by Mermilliod et al. (1996) who studied the red giant content of this cluster and found $m-M=11^{\mathrm{m}} 50, E(B-V)=0.29$ and $\log t=8.65$. In contrast to the above, the WEBDA catalogue (http://obswww. unige.ch/webda) lists $m-M=$ 11. $67, E(B-V)=0 . \cdot 302, \log t=8.540$, however, the differences of the quoted values do not exceed the expected uncertainties. To our knowledge, there has been no variable star survey in the literature despite the relative proximity of the cluster. This neglect might be caused by the strong concentration of stars that makes it difficult to separate individual stars. 
Table 1. The journal of observations.

\begin{tabular}{lcccl}
\hline Date & $\begin{array}{c}B \\
\text { frames }\end{array}$ & $\begin{array}{c}V \\
\text { frames }\end{array}$ & $\begin{array}{c}R \\
\text { frames }\end{array}$ & $\begin{array}{l}\text { length } \\
(\mathrm{min})\end{array}$ \\
\hline 1999 Dec. 27/28 & - & - & 120 & 190 \\
1999 Dec. 29/30 & 3 & 3 & 30 & 100 \\
1999 Dec. 30/31 & - & - & 187 & 280 \\
2000 Jan. 28/29 & - & - & 145 & 340 \\
2000 Jan. 30/31 & - & - & 43 & 90 \\
2000 Jan. 31/Feb. 01 & - & - & 58 & 150 \\
2000 Feb. 01/02 & - & - & 75 & 170 \\
\hline Total: & 3 & 3 & 658 & 1320 \\
\hline
\end{tabular}

\section{Observations and data analysis}

CCD BVR photometric observations were carried out on 7 nights between December 27, 1999 and February 1, 2000 at the Piszkéstető Station of the Konkoly Observatory (Hungary). The instrument used was the 60/90/180 cm Schmidt telescope, equipped with a Photometrics AT200 CCD $(1536 \times 1024$ pixels, KAF-1600 chip with UVcoating). The angular resolution was $1^{\prime \prime} 1 /$ pixel, yielding a field of view of $29^{\prime} \times 18^{\prime}$. Time-series observations were made through an $R$ filter (60 and 120 s exposures), while three $B$ and $V$ filtered frames were also taken on December 29, 2000 in order to obtain colour information on the detected stars (20 s, $60 \mathrm{~s}$ and $300 \mathrm{~s}$ exposures). The choice of using an $R$ filter in the time-series observations was due to an unfortunate technical difficulty, that made other filters unavailable on other nights. The full observing $\log$ is given in Table 1.

The basic image reduction was carried out using the standard tasks in IRAF. The flat field corrections made use of sky-flat images taken during the evening twilight. We performed psf-photometry with the daophot package of IRAF using the Moffat point-spread function, which turned out to be well suited for fitting the slightly distorted stellar profiles (caused by the optical layout of the Schmidt system). The magnitudes were calculated relative to the ensemble mean of a selected set of stars, with an arbitrarily chosen zero-point. Typically, we detected about 3000 stars on the $R$ frames. The co-ordinate system of each frame was corrected for two-dimensional shift, rotation and optical distortions, relative to a master frame. The master frame is the one which contained the largest number of stars, and is likely to contain every stars detected in the series. Although there is still some small chance of loosing a few stars that were undetected on the given starting image, the overwhelming majority of the stars are properly identified. We could identify 2530 stars on at least 20 frames. The last step in data filtering was to reject those stars that were closer than 30 pixels to the image edges. The finally adopted data set contains 2323 individual light curves. We note, that $R$ data on Dec. 29/30, 1999 (30 points) were excluded from the photometric analysis because of their larger noise level.
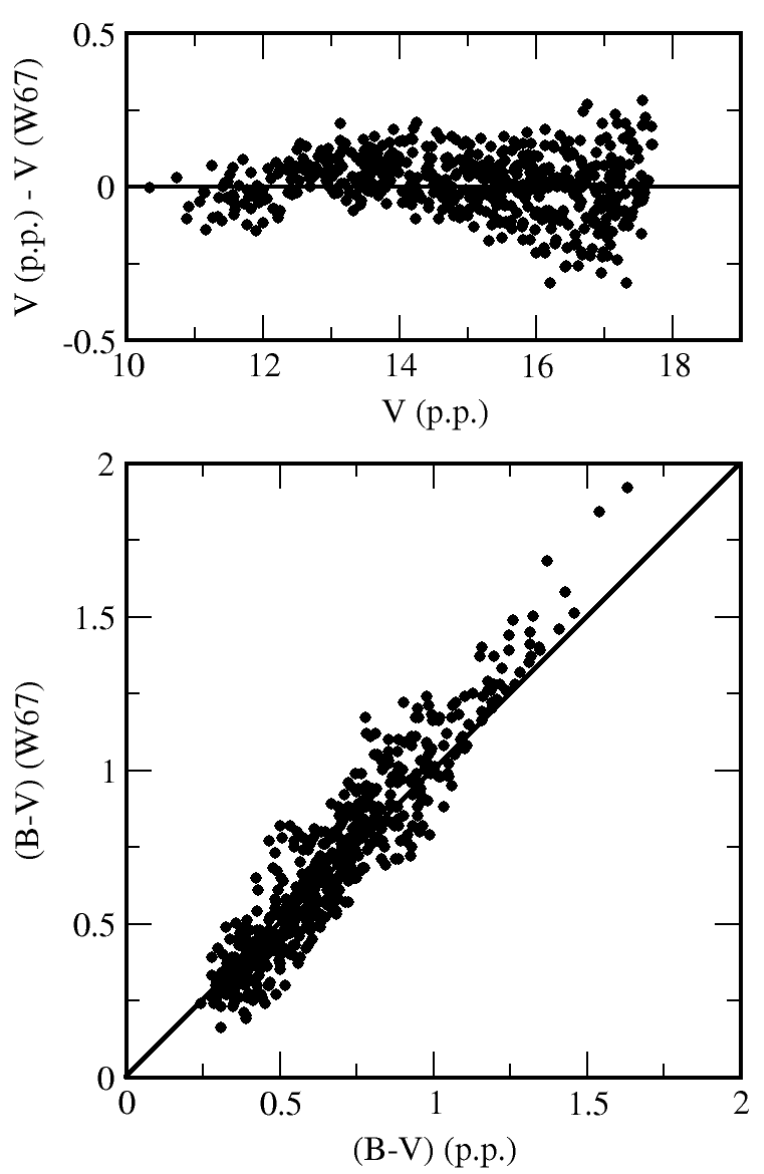

Fig. 1. A comparison of the $V$ magnitudes and $B-V$ colours obtained in this study with data by West (1967). The diagrams are based on 580 common stars. The solid lines denote equality.

The weather conditions were not photometric when the three-colour observations were obtained, therefore, we could not perform standard all-sky photometry in order to standardise the observed colours and magnitudes. Thus, we have tied our data to the photoelectric $U B V$ photometric secondary standards in the cluster taken from Table 2 in West (1967). The colour-dependent transformation coefficients $\epsilon$ and $\mu(\Delta V=\Delta v+\epsilon \Delta(B-V)$, $\Delta(B-V)=\mu \Delta(b-v))$ were determined by observing the standard sequence of Schild (1983) in the open cluster M $67(\epsilon=0.10 \pm 0.03, \mu=0.83 \pm 0.03)$. This $\mu$ value indicates filters or a detector quite far from the standard, fortunately, the standard star observations gave well-defined linear transformation equations with no indication of second-order terms. We have compared the resulting $V$ and $B-V$ data with those of West (1967) and can unambiguously identify 580 stars common to both sets which are plotted in Fig. 1. There are some slight systematic differences between the $V$ data which are characterized by the mean deviation of $V$ (p.p.) $-V(\mathrm{~W} 67)$ and the rms around that mean in certain magnitude ranges. The results are: for $V=10-12$ mag the mean deviation is -0 m 032 with an rms 0.043 ; for $V=12-14$ mag $+0{ }^{\mathrm{m}} 044,0{ }^{\mathrm{m}} 049$; for $V=14-16 \mathrm{mag}+0{ }^{\mathrm{m}} 015,0.067$; for $V=16-18 \mathrm{mag}-0{ }^{\mathrm{m}} 013,0{ }^{\mathrm{m}} 098$. The colour data are also 


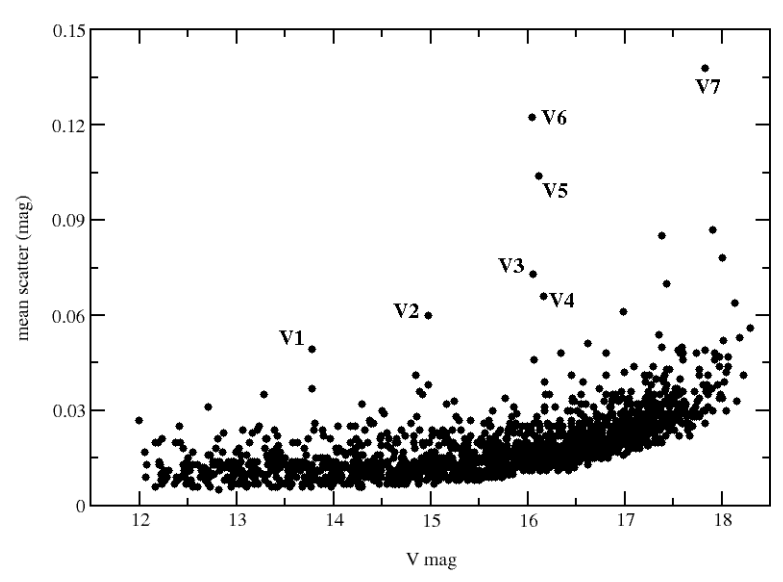

Fig. 2. The scatter of individual stars as a function of $V$ magnitude. Note the absence of the brightest stars (between $\left.V=11^{\mathrm{m}} 0 \ldots 12^{\mathrm{m}} 0\right)$ in the cluster field, which were saturated in the time series data observations.

affected by these systematic differences. The largest deviations occur for the reddest stars, our values are slightly bluer than those of West (1967). This inconsistency is due to the fact that red stars beyond $B-V \approx 1$. 50 were essentially extrapolated with the standard transformations. The two reddest photoelectric standards in Table 2 of West (1967) have $B-V 1$. 626 and 1.559 and such red stars are very likely variable stars (Jorissen et al. 1997). In summary, the uncertainty of standard $V$ and $B-V$ data changes from 0.03 to 0.10 , depending on the colour and apparent brightness of the stars. Since we could not standardise the $R$-band data, their actual values contain the arbitrary zero-point.

The search for variable stars was performed by checking the light curve statistics and by visual inspection of the suspected variable time-series data. We have calculated the scatter of individual light curves for every star (in sense of $\left.\left\langle m_{\mathrm{obs}}-\left\langle m_{\mathrm{obs}}\right\rangle\right\rangle\right)$ ) and it is plotted as a function of $V$ magnitude in Fig. 2. The identified new variables are also labelled. The main ridge helped us to characterise the accuracy of the brightness measurements, which changed between $0{ }^{\mathrm{m}} 01-0 \mathrm{~m} 06$ over a range of $\sim 6 \mathrm{mag}$. As can be seen in Fig. 2, besides the strongly deviant points, there is a group of slightly deviant points over the main concentration. We have checked all of them and found that those stars are in the most crowded fields in the cluster, where the applied psf-photometry may suffer from larger photometric errors. That is why all of the data with suggested larger scatter was inspected visually. Seven stars remained as unambiguously variable stars and these are identified in the $2 \times 2^{\prime}$ finding charts in Fig. 3 .

The light curves of new variable stars were further analysed to search for possible periodicities. For this, we performed conventional Fourier analysis of the data with Period98 (Sperl 1998). However, in case of eclipsing binaries, the phase dispersion minimisation (PDM - Stellingwerf 1978) is more suitable for inferring periodicities. Thus, PDM spectra enabled an independent check of the results. Original data are available electronically at the CDS via anonymous ftp to cdsarc.u-strasbg.fr (130.79.128.5) or via http://cdsweb.u-strasbg.fr/cgi-bin/qcat?J/A+A/376/561

\section{Results}

The analysis consisted of the following steps: i) construction of the colour-magnitude (CM) diagram and physical parameter estimation of the cluster; ii) variable star classification based on the light curve shapes; iii) period determination for stars with well-sampled light curves; iv) membership investigation (considering the location of new variables on the CM diagram and using various period-colour-luminosity relations that are valid either for W UMa or $\delta$ Scuti stars).

\subsection{The colour-magnitude diagram}

The physical parameters of the cluster have been estimated by a isochrone fitting of the CM diagram. Since we could not determine the interstellar reddening independently this parameter has been also included in the fitting procedure. The isochrones were taken from Bertelli et al. (1994) and the initial parameters were adopted from Mermilliod et al. (1996). The result is shown in Fig. 4, where the CM diagram is plotted with the two closest isochrones $z=0.02$. Note the strong field star contamination, which is caused by the position of the cluster in the galactic plane. The overall morphology is well reproduced, except that the models are too red for the red giant stars. Note that this diagram is essentially the same as Fig. 11 in Mermilliod et al. (1996), the only difference is that our graph is based on a much larger data set which extends to $\sim 2$ mag below the faint limit in Mermilliod et al. (1996). Therefore, the resulting parameters are very similar to those of Mermilliod et al. (1996): the colour excess is $E(B-V)=0.29 \pm 0.03$, the distance modulus is $m-M=11^{\mathrm{m}} 48 \pm 0.13$ and $\log t=8.65$. These values were adopted in this paper.

\subsection{The new variable stars}

In the following section we describe the new variables and their light variations. Eclipsing binaries and pulsating variables are discussed separately. Thanks to the moderately good phase coverage, five of the seven new variables have unambiguous periods and classification. The remaining two stars have characteristic light variation of longperiod detached eclipsing binaries, i.e. long constancy interrupted by short fadings. We have given the stars the V1...V7 designations, where the numbers increase with the average magnitude. Their basic data are summarised in Table 2.

\subsubsection{Eclipsing binaries}

V1: There was only one fading of 0.24 on JD 2451572. Unfortunately, we did not observe the subsequent brightening, thus no epoch of minimum could be determined. 

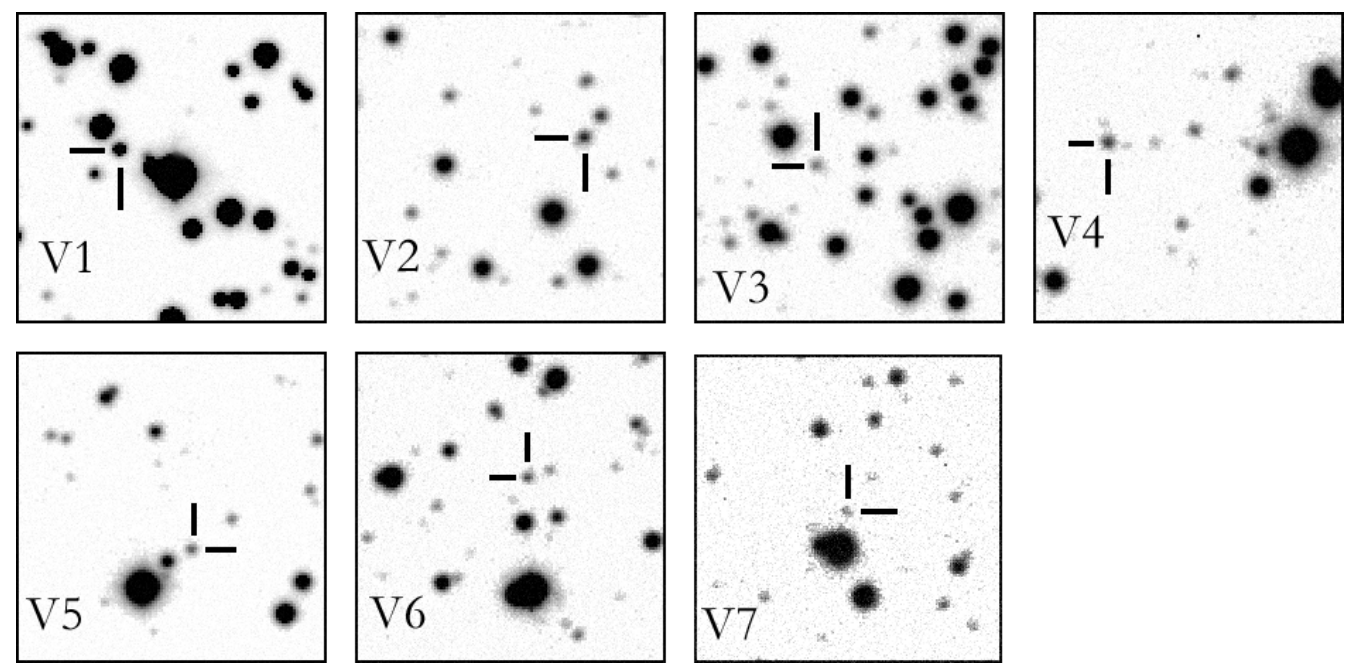

Fig. 3. Finding charts for the newly discovered variable stars. Each frames are $2 \times 2^{\prime}$ wide, north is up, east is to the left.

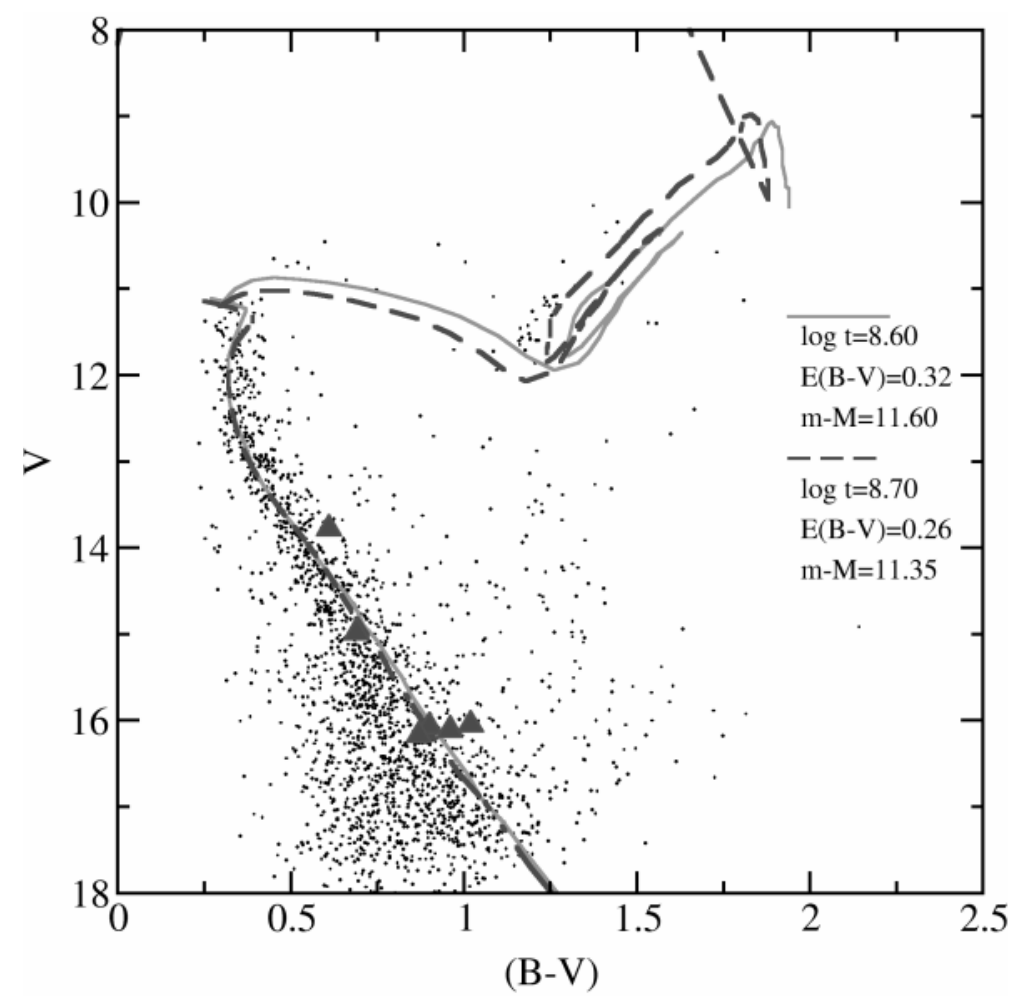

Fig. 4. The colour-magnitude diagram of M 37 with two isochrones (solar composition) taken from Bertelli et al. (1994). The morphology is well reproduced, although the red giant models are slightly too red. The positions of new variable stars are marked with the up triangles. The brightest one is V1 (type EA:), the next one is V2 (EA:), while the four variables at $V \approx 16$ mag are V4 (EW), V3 (EW), V5 (DSCT/RRc) and V6 (DSCT) starting from the bluest one. V7 is not presented in this diagram because it was not detected on the $B$ frames.

The first night of observations revealed an ambiguous brightening, which may be the ascending branch of a primary minimum. The light curves are plotted in panel (a) of Fig. 5. The larger scatter in subpanel a3 was caused by the bad seeing which increased the light contamination from the close bright neighbours (see its finding chart in Fig. 3).

V2: We observed a 0.23 deep minimum on JD 2451540 which occured at $\operatorname{HJD}(\min )=2451540.518$. The other observations showed no significant variation, thus no period could be determined. The light curves are shown in panel (b) of Fig. 5 .

V3: The amplitude is 0.32 , while the inferred period is $0.4224 \pm 0.0001$ days. The light curve shape is very characteristic for a contact binary. The phase diagram of the whole light curve is shown in Fig. 6. We note the slightly different maxima that might be a hint of a spotted stellar surface. 
V1

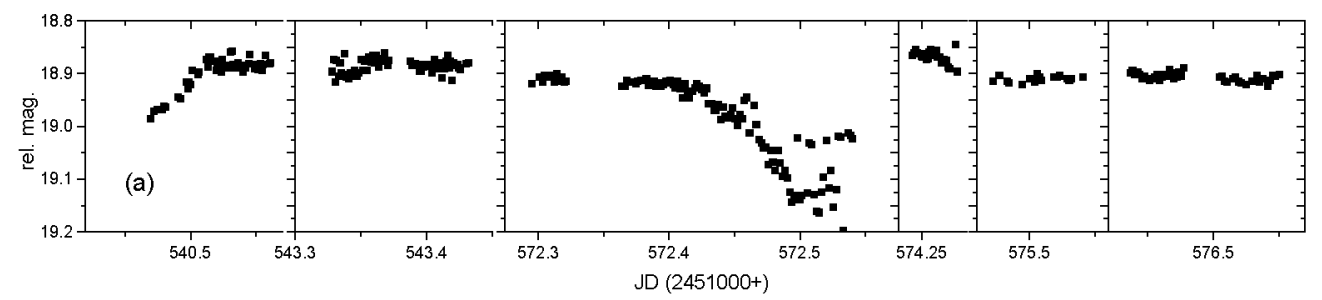

V2

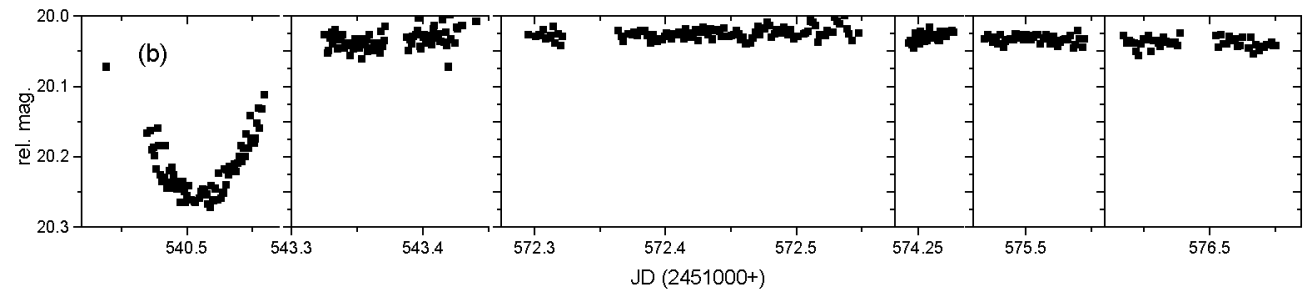

Fig. 5. The light curves for two new suspected Algol-type eclipsing binaries.
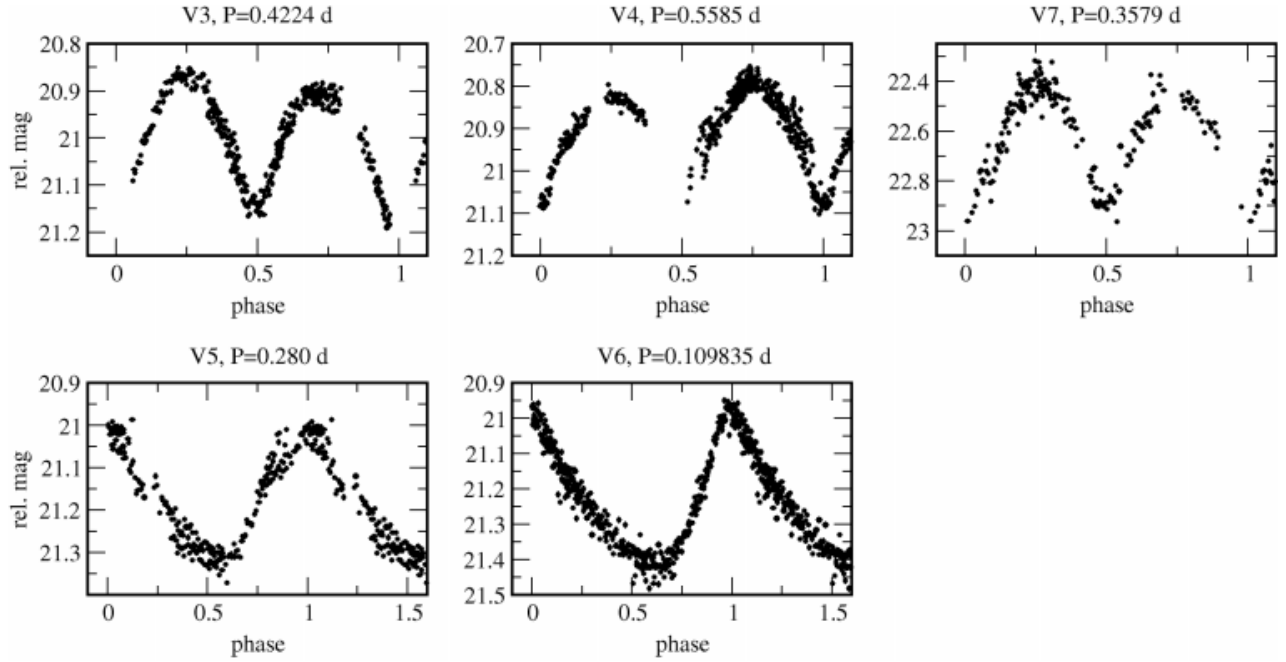

Fig. 6. The phase diagrams for V3...V7.

V4: The light curve is typical of an EW-type star with an amplitude of $0 .{ }^{\mathrm{m}} 33$. There are interesting humps on the light curve suggesting again the possibility of photometric surface inhomogeneities. The inferred period is $0.5585 \pm$ 0.0001 days. The phase diagram is presented in Fig. 6 .

V7: This is the faintest variable star detected by us in the cluster, and that is why its light curve contains only 185 points (it has been lost from many frames by the star detection algorithm). The observed amplitude is 0.55 , while the period is about $0.3579 \pm 0.0001$ days. The phase diagram shows a typical W UMa-type variation (Fig. 6).

\subsubsection{Pulsating variables}

V5: The short-period asymmetric light curve with an amplitude of 0.32 is typical of pulsating stars. The period is $0.2800 \pm 0.0005$ days. Since it is located very close to the edge of the field, only 5 nights of data were analysed and their phase diagram is shown in Fig. 6.
V6: The light variation is that of a monoperiodic highamplitude $\delta$ Scuti-star $(P=0.109835 \pm 0.000005$ days, $A=0.45)$. The phase diagram (Fig. 6) also suggests a remarkably repetitive light change.

We have also studied the possible multiperiodic nature of V5 and V6 by a standard Fourier analysis of their light curves. After prewhitening with the main frequencies (and their harmonics), the residual frequency spectra did not show any significant peak. However, because of the relatively short span of observations we cannot firmly conclude the strict monoperiodicity of these variable stars.

\subsection{Cluster membership of the variable stars}

We have marked the locations of the variable stars on the CM diagram in Fig. 4 and must stress that these positions, based on a single-epoch observation, are uncertain to $\sim \pm 0.2$, as both the $B-V$ colour and $V$ magnitude change over the time. This effect is larger for the pulsating variables, which usually have more variable colour indices 
Table 2. The basic data of new variables.

\begin{tabular}{|c|c|c|c|c|c|c|c|c|}
\hline Star & $\alpha_{2000}$ & $\delta_{2000}$ & $\langle V\rangle$ & $\langle B-V\rangle$ & $P(\mathrm{~d})$ & $A$ (mag) & $\operatorname{Epoch}(\mathrm{s})$ & Type \\
\hline V1 & $05^{\mathrm{h}} 52^{\mathrm{m}} 20^{\mathrm{s}} \cdot 42$ & $+32^{\circ} 33^{\prime} 19^{\prime \prime} .5$ & $13^{m} 78$ & 0.61 & - & - & - & EA: \\
\hline $\mathrm{V} 2$ & $05^{\mathrm{h}} 52^{\mathrm{m}} 16^{\mathrm{s}} .60$ & $+32^{\circ} 28^{\prime} 14^{\prime \prime} .9$ & $14^{\mathrm{m}} 98$ & $0 .{ }^{\mathrm{m}} 69$ & - & - & 2451540.518 & EA: \\
\hline V3 & $05^{\mathrm{h}} 52^{\mathrm{m}} 33^{\mathrm{s}} .03$ & $+32^{\circ} 32^{\prime} 41^{\prime \prime} .7$ & 16.07 & $0 .{ }^{\mathrm{m}} 90$ & $0.4224(1)$ & 0.31 & 2451575.5083 (II) & EW \\
\hline V4 & $05^{\mathrm{h}} 52^{\mathrm{m}} 53^{\mathrm{s}} .26$ & $+32^{\circ} 33^{\prime} 01^{\prime \prime} .2$ & 16.17 & 0.87 & $0.5585(1)$ & 0.33 & 2451576.456: (I) & EW \\
\hline V5 & $05^{\mathrm{h}} 53^{\mathrm{m}} 00^{\mathrm{s}} .63$ & $+32^{\circ} 24^{\prime} 50^{\prime \prime} 8$ & $16^{\mathrm{m}} 11$ & $0 .{ }^{\mathrm{m}} 96$ & $0.2800(5)$ & 0.32 & 2451576.500: & DSCT \\
\hline V6 & $05^{\mathrm{h}} 51^{\mathrm{m}} 50.55$ & $+32^{\circ} 32^{\prime} 34^{\prime \prime} .4$ & $16 \cdot 05$ & 1. 02 & $0.109835(5)$ & 0.45 & 2451540.5367 & DSCT \\
\hline V7 & $05^{\mathrm{h}} 52^{\mathrm{m}} 39^{\mathrm{s}} .32$ & $+32^{\circ} 36^{\prime} 30^{\prime \prime} .9$ & $17^{\mathrm{m}} 83$ & - & $0.3579(1)$ & 0.55 & 2451574.262: (I) & EW \\
\hline
\end{tabular}

than eclipsing stars. The locations of the eclipsing binaries are consistent with the stars being members of the cluster, as they scatter around the main sequence. This can be further studied for V3 and V4 with the period-colourluminosity relation published by Rucinski \& Duerbeck (1997). Their Eq. (5a) $\left(M_{V}=-4.44 \log P+3.02(B-\right.$ $\left.V)_{0}+0.12\right)$ predicts $M_{V}(\mathrm{~V} 3)=3{ }^{\mathrm{m}} 62$ and $M_{V}(\mathrm{~V} 4)=3 .{ }^{\mathrm{m}} 00$ which is about 1-1.5 mag brighter than the calculated absolute magnitudes using the cluster's distance modulus. Note, that when calculating the absolute magnitude, the unreddened colour should be estimated, e.g., with help of the period-colour diagram of W UMa-type systems (Fig. 2 in Rucinski \& Duerbeck 1997). However, the above quoted difference does not exlude the membership of V3 and V4, because even the calibrating data set of Rucinski \& Duerbeck (1997) contained stars with similarly large deviations.

Contrary to the eclipsing binaries, the pulsating variables are too red and too faint to be cluster members. The $\delta$ Scuti instability strip of the H-R diagram (Breger 2000; Rodríguez \& Breger 2001) is in a bluer and much brighter region than that containing V5 and V6 (e.g. the apparent brightness range of the instability strip of $\mathrm{M} 37$ is about 11. $\left.5-14^{\mathrm{m}} 0\right)$.

Petersen \& Christensen-Dalsgaard (1999) discussed the Hipparcos period-luminosity relation for highamplitude $\delta$ Scuti stars and concluded that quite good absolute magnitudes $( \pm 0 . \mathrm{m} 1)$ can be inferred from the resulting PL-relation. Their Eq. (4) $\left(M_{V}=-3.75 \log P-1.969\right)$ predicts $M_{V}(\mathrm{~V} 5)=0.1$ and $M_{V}(\mathrm{~V} 6)=1.6$. Both values suggest the stars are much further away than M 37. Unfortunately, farther means redder and the corresponding higher colour excesses cannot be properly estimated.

Furthermore, the period and light curve shape of V5 also suggests the possibility of being a field RRc star instead of high-amplitude $\delta$ Scuti star. In that case its absolute magnitude should be about $0.5(\log L \approx 1.7$, e.g. Kolláth et al. 2000), which yields to a distance modulus $m-M(\mathrm{~V} 5)=15{ }^{\mathrm{m}} 5$. The RRc assumption gives a mean $(B-V)_{0}$ colour of $0 .{ }^{\mathrm{m}} 2-0{ }^{\mathrm{m}} 4$ (e.g. Lee \& Carney 1999) resulting in $E(B-V) \approx 0$. 7 . On the other hand, there are a few longer period $\delta$ Scuti stars known with periods between 0.25 and 0.3 days (Rodríguez \& Breger 2001), that could be evolved massive $\delta$ Scuti variables. Thus, V5 can be either a new field RRc star or a high-amplitude, longer period $\delta$ Scuti star. In any case, neither V5 nor V6 seems to be physical a member of M 37 .

\section{Summary}

We have presented the first variable star survey of the rich open cluster and have discovered seven new variable stars, two pulsating and five eclipsing. Epochs and periods are determined for three W UMa-type systems and two pulsating stars. The eclipsing binaries are probably cluster members, while the pulsating stars are background objects lying much further away than M 37. V5 might be either a high-amplitude $\delta$ Scuti or a field RRc star, while V6 is classified unambiguously as high-amplitude $\delta$ Scuti star. More observations are needed to determine periods for $\mathrm{V} 1$ and $\mathrm{V} 2$, while it is an interesting question whether the observed light curve shapes of the contact binaries are stable over a longer period. All of the three W UMa systems have light curves with different consecutive maxima suggesting surface inhomogeneities that can be caused by stellar spot activity.

In addition to the variable star survey, a new colourmagnitude diagram has been constructed which is based on about 2300 stars. Since the cluster lies very close to the galactic plane, the field star contamination is fairly strong. We have determined independently the cluster's main physical parameters by fitting isochrones taken from Bertelli et al. (1994). The best fits yield $E(B-V)=$ $0.29 \pm 0.03, m-M=11^{\mathrm{m}} 48 \pm 0.13$ and $\log t=$ 8.65. In more convenient units, the cluster's age is about $450 \times 10^{6}$ years and the distance to M 37 is about $1300 \mathrm{pc}$. These parametes are in good agreement with those available in the literature.

Note added in proofs: After the acceptance of the paper, S. Rucinski (personal communication) pointed out that V3 and V4 are more likely non-member field contact binaries. This is based on their deviation from the periodcolor-luminosity relation of W UMa-stars and the estimated number of projected contact binaries in the galactic field. For the given angular area of our search and the distance of the cluster, one would expect approximately 2-3 projected field contact binaries from the frequency-ofoccurence distribution of W UMa-type stars in the galactic disk (Rucinski 1998). Nevertheless, this point does not affect other conclusions of the paper. 
Acknowledgements. This project has been supported by FKFP Grant 0010/2001, OTKA Grant \#T032258, "Bolyai János" Research Scholarship of LLK from the Hungarian Academy of Sciences and Szeged Observatory Foudation. The warm hospitality of the staff of the Konkoly Observatory and their provision of telescope time is gratefully acknowledged. Thanks are due to the referee, Dr. S. Frandsen, whose suggestions led to significant improvement of the paper. The authors also acknowledge careful reading of the manuscript by C. Lloyd. The NASA ADS Abstract Service was used to access data and references. This research has made use of the SIMBAD database, operated at CDS-Strasbourg, France.

\section{References}

Bertelli, G., Bressan, A., Chiosi, C., et al. 1994, A\&AS, 106, 275

Breger, M. 2000, in Delta Scuti and Related Stars, Reference Handbook and Proc. of the 6th Vienna Workshop in Astrophysics, ed. M. Breger, \& M. Montgomery, ASP Conf. Ser., 210, 3

Frandsen, S., \& Arentoft, T. 1998, A\&A, 333, 524
Jefferys III, W. H. 1962, AJ, 67, 532

Jorissen, A., Mowlavi, N., Sterken, C., \& Manfroid, J. 1997, A\&A, 324, 578

Kałużny, J., \& Rucinski, S. M. 1993, ASP Conf. Ser., 53, 164

Kolláth, Z., Buchler, J. R., \& Feuchtinger, M. 2000, ApJ, 540, 468

Lee, J.-W., \& Carney, B. W. 1999, AJ, 117, 2868

Lyngå, G. 1987, Fifth catalogue of cluster parameters, Strasbourg

Mermilliod, J.-C., Huestamendia, G., del Rio, G., \& Mayor, M. 1996, A\&A, 307, 80

Petersen, J. O., \& Christensen-Dalsgaard, J. 1999, A\&A, 352, 547

Robin, A. 1982, A\&AS, 50, 251

Rodríguez, E., \& Breger, M. 2001, A\&A, 366, 178

Rucinski, S. M., 1998, AJ, 116, 2998

Rucinski, S. M., \& Duerbeck, H. W. 1997, PASP, 109, 1340

Schild, R. E. 1983, PASP, 95, 1021

Sperl, M. 1998, Comm. Astr. Seis., 111

Stellingwerf, R. F. 1978, ApJ, 221, 661

Upgren, A. R. 1966, AJ, 71, 736

West, F. R. 1967, ApJS, 14, 359 